\title{
Characterization of power induced heating and damage in fiber optic probes for near-field scanning optical microscopy
}

\author{
Nicholas E. Dickenson, Elizabeth S. Erickson, Olivia L. Mooren, and Robert C. Dunn ${ }^{\text {a) }}$ \\ Department of Chemistry, University of Kansas, Multidisciplinary Research Building, 2030 Becker Drive, \\ Lawrence, Kansas 66047
}

(Received 1 March 2007; accepted 20 April 2007; published online 16 May 2007)

\begin{abstract}
Tip-induced sample heating in near-field scanning optical microscopy (NSOM) is studied for fiber optic probes fabricated using the chemical etching technique. To characterize sample heating from etched NSOM probes, the spectra of a thermochromic polymer sample are measured as a function of probe output power, as was previously reported for pulled NSOM probes. The results reveal that sample heating increases rapidly to $\sim 55-60{ }^{\circ} \mathrm{C}$ as output powers reach $\sim 50 \mathrm{nW}$. At higher output powers, the sample heating remains approximately constant up to the maximum power studied of $\sim 450 \mathrm{nW}$. The sample heating profiles measured for etched NSOM probes are consistent with those previously measured for NSOM probes fabricated using the pulling method. At high powers, both pulled and etched NSOM probes fail as the aluminum coating is damaged. For probes fabricated in our laboratory we find failure occurring at input powers of $3.4 \pm 1.7$ and $20.7 \pm 6.9 \mathrm{~mW}$ for pulled and etched probes, respectively. The larger half-cone angle for etched probes $\left(\sim 15^{\circ}\right.$ for etched and $\sim 6^{\circ}$ for pulled probes) enables more light delivery and also apparently leads to a different failure mechanism. For pulled NSOM probes, high resolution images of NSOM probes as power is increased reveal the development of stress fractures in the coating at a taper diameter of $\sim 6 \mu \mathrm{m}$. These stress fractures, arising from the differential heating expansion of the dielectric and the metal coating, eventually lead to coating removal and probe failure. For etched tips, the absence of clear stress fractures and the pooled morphology of the damaged aluminum coating following failure suggest that thermal damage may cause coating failure, although other mechanisms cannot be ruled out. (C) 2007 American Institute of Physics. [DOI: 10.1063/1.2740133]
\end{abstract}

\section{INTRODUCTION}

Several techniques have been developed that enable optical measurements to be conducted with very high spatial resolution. One approach, aperture based near-field scanning optical microscopy (NSOM), uses aluminum coated, tapered, single mode optical fiber probes to deliver light down to the nanometric dimension. By scanning these probes within nanometers of a sample surface, high resolution optical and topographical information is simultaneously recorded. ${ }^{1-3}$ This technique has been used extensively to study lipid membranes, thin films, liquid crystals, and solid-state materials. ${ }^{1,4-6}$

Much work has been devoted to the characterization and further development of the probes used in NSOM to deliver light. As light travels down the taper region of a NSOM probe towards the aperture, light is lost into the surrounding metal coating of the probe. This leads to the two related phenomena of tip heating and failure, the characterization of which have been problematic due to the small size of the probe. ${ }^{3,7,8}$ However, understanding both of these processes is critical for the application of NSOM to heat sensitive samples and for further development of NSOM probes.

Fiber optic NSOM probes are generally fabricated using either the heating and pulling method or the chemical etching

\footnotetext{
a) Author to whom correspondence should be addressed.
}

approach. Both methods produce tapers in the fibers that terminate at small tips that, once coated with a metal, produce small apertures suitable for NSOM measurements. $6,9,10$ The main difference in probes fabricated with the two approaches is in the cone angle of the probe. The chemical etching technique can produce much larger cone angles that result in highly efficient NSOM probes compared to those fabricated with the heating and pulling method. ${ }^{11,12}$ Etched probes also have increased optical transmission which leads to a number of advantages such as decreased acquisition times for spectroscopic analysis including fluorescence and Raman measurements as well as making high density, nanometric readwrite technology feasible. ${ }^{6,13}$ The increased efficiency of etched probes may also lead to differences in the heating characteristics and failure mechanism compared to pulled NSOM probes.

Early studies of NSOM tip heating found a significant elevation in probe temperature as the input power was increased. ${ }^{8,14-17}$ Using a thermocouple positioned along the taper region of the probe tens of microns from the aperture, temperatures as high as $470{ }^{\circ} \mathrm{C}$ were measured. ${ }^{8}$ Since the temperature was recorded along the probe taper, however, it remained unclear how much heating the sample actually experienced under the aperture of the NSOM probe. Recent studies using thermochromic samples have provided a more direct view of sample heating during NSOM measurements. ${ }^{18}$ 
We recently reported results using the "two-color" emission from a thermochromic polymer consisting of perylene and $N$-allyl- $N$-methylaniline (NA) to characterize sample heating under pulled NSOM probes. These ratiometric measurements found that as power increases, sample heating quickly rises and levels off at a maximum temperature of $\sim 65{ }^{\circ} \mathrm{C}$. As output power was further increased to $200 \mathrm{nW}$, no change was observed in the sample heating. The leveling off in sample heating was ascribed to heat induced expansion of the NSOM probe which increases the distance between the probe aperture and the sample surface. ${ }^{15,18}$

Here we use a similar approach to characterize sample heating with chemically etched NSOM probes. It has been suggested that the increased efficiencies of these probes may reduce sample heating. ${ }^{19,20}$ On the contrary, our measurements show that these probes have nearly identical sample heating profiles with output power as NSOM probes fabricated using the pulling method. While the heating is similar, we find that the power at which tip failure occurs is much higher for etched tips than for pulled probes. NSOM probe failure is accompanied by a dramatic increase in optical transmission from the probe as the subwavelength diameter aperture is destroyed. The failure is known to be a result of damage to the metal coating near the apex, though the mechanism is currently unclear and has been attributed to either fracturing of the metal coating due to shear stresses or a melting of the metal coating itself. ${ }^{3}$

To compare tip failure from both etched and pulled probes, both probe types were monitored as a function of input power until they reached failure. High resolution im-

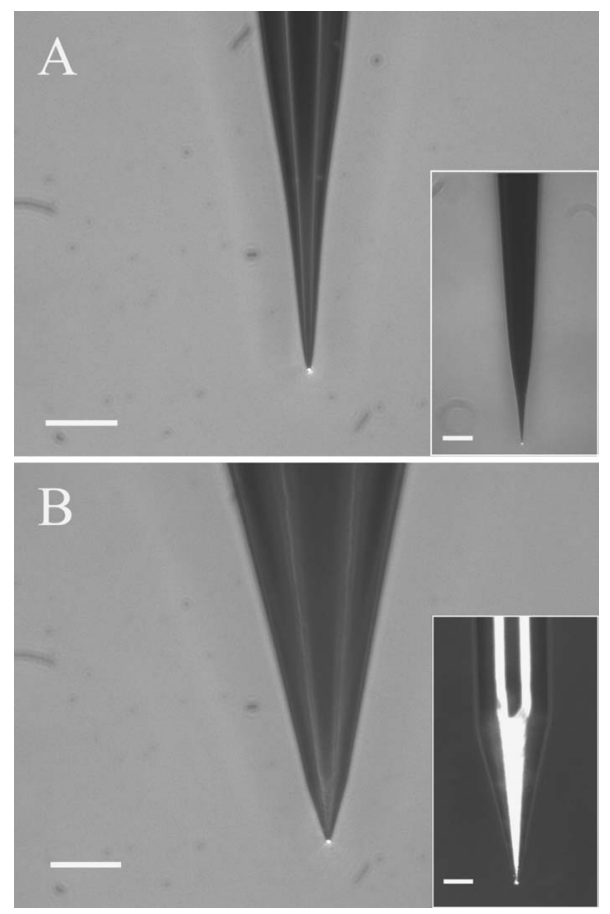

FIG. 1. Magnified views of fiber optic NSOM probes fabricated with the (A) pulling and (B) chemical etching methods (scale bars are $30 \mu \mathrm{m}$ ). Each probe has a well-defined aperture indicated by the single spot of light emerging from the end of the tip. NSOM probes fabricated with the etching technique (B) have much larger cone angles which increase the efficiency of light delivery. The insets show expanded views where the scale bars are $50 \mu \mathrm{m}$. aging indicates that the failure of both probe types results in a compromised metal coating near the apex, though the mechanisms of failure appear different. The pulled probes failed through a mechanism involving stress fractures in the metal coating caused by the differential thermal expansion of the dielectric and surrounding metal coating. The etched probes, however, appear to fail as a result of melting of the aluminum coating.

\section{MATERIALS AND METHODS}

\section{A. Fabrication of NSOM probes}

Etched NSOM probes were manufactured by the Turner method in which stripped and cleaved single mode fiber optic (460-HP, Nufern) is placed into a Teflon vial containing a $49 \%$ solution of hydrofluoric acid (HF). ${ }^{12,21}$ The acid is covered with a thin layer of silicon oil in order to protect the rest of the fiber from the HF vapors. The HF forms a meniscus at the fiber and etches away the fiber, thus forming the taper and apex of the probe. Using a homebuilt vacuum deposition chamber, the probes are then coated with $\sim 200 \mathrm{~nm}$ of aluminum in order to confine light and guide it to the aperture. Pulled NSOM probes were produced by mechanically heating and pulling a single mode fiber optic with a Sutter P-2000 micropipette puller. The taper of the pulled fibers was coated with $\sim 50-100 \mathrm{~nm}$ of aluminum. The details of the fabrication process are discussed elsewhere. ${ }^{22}$

\section{B. Temperature measurements of etched probes}

The temperature sensitive perylene-NA polymer was synthesized as previously described. ${ }^{23} \mathrm{~A}$ thin film of the perylene-NA polymer was coated onto a glass coverslip. The NSOM probes were held in feedback nanometers from the surface of the polymer with a homebuilt shear-force head controlled with a Nanoscope IIIa controller (Veeco Instruments) as previously described. ${ }^{24}$ The emission spectra of the polymer following excitation at $405 \mathrm{~nm}$ (Power Technology) were collected (Ziess 40X, 1.30 NA) and sent to a spectrograph (SpecrtaPro 300i, Acton Research Corporation) coupled to a CCD camera (DU420-BV, Andor Technology).

\section{Imaging of NSOM probe failure}

To visualize the mechanism of tip failure, both pulled and etched aluminum coated NSOM probes were imaged with high resolution in a focused ion beam (FIB) instrument (Micrion 9000) outfitted with a custom feedthrough flange that allowed light to be coupled into the probe. High resolution images of the NSOM probe were collected as a function of laser power (488 nm line, Coherent ENTCII-621 argon ion laser) coupled into the probe. Images of the tips were taken as the power into the probe was gradually increased until they ultimately failed.

\section{RESULTS AND DISCUSSION}

\section{A. Sample heating from etched NSOM probes}

Figure 1 compares phase contrast images of NSOM probes fabricated (A) using the heating and pulling method and (B) the chemical etching technique. The sides of the 
taper have been coated with $\sim 100 \mathrm{~nm}$ of aluminum to confine the light until it exits the aperture as a well-defined, single spot of light as seen in Fig. 1. As has been well documented, etching allows for much larger cone angles in the taper region of the probe which increases the efficiency of light delivery. ${ }^{3}$ The half-cone angle for the pulled probe in Fig. 1(a) is $6^{\circ}$, compared with $15^{\circ}$ for the etched tip shown in Fig. 1(b). The overall shapes of the probes are clearly seen in the insets of Fig. 1. While the entire taper region of the etched probe, starting from the $125 \mu \mathrm{m}$ width of fiber optic, is visible, typical taper regions in pulled probes extend over $\sim 500-800 \mu \mathrm{m}$.

As light travels down the taper region toward the NSOM probe aperture, the number of guided modes continually decreases. As the last guided mode reaches the cutoff diameter, the energy decays exponentially towards the aperture of the probe which accounts for the low throughput of NSOM probes. ${ }^{6,25}$ Because of the larger cone angle, etched probes support guided modes closer to the probe aperture and therefore provide much higher throughput than pulled NSOM probes. $^{3,6,26}$ The higher throughput efficiencies of etched NSOM probes has led to speculation that sample heating may be reduced, making chemically etched probes more amenable for use with temperature sensitive samples. ${ }^{19,20}$

Previously, we used a thermochromic polymer to characterize the sample heating experienced directly from pulled NSOM probes. ${ }^{18}$ The inset in Fig. 2 shows the temperature dependent emission spectra of the perylene-NA polymer normalized to the $475 \mathrm{~nm}$ peak. Ratiometric measurements of the emission intensities from the 475 and $510 \mathrm{~nm}$ peaks provide an intrinsic measure of sample heating that is independent of excitation power. ${ }^{18,23}$ This provides an ideal sample for quantifying sample heating as a function of output power from NSOM probes.

Figure 2 shows a plot of sample temperature versus probe output power collected for several etched NSOM probes. The shear-force feedback method was used to hold the probes within nanometers of the surface for all measurements. As shown in Fig. 2, the sample temperature quickly rises with probe output power. As the output power from the probe reaches $50-75 \mathrm{nW}$, the sample heating levels off at approximately $55-60{ }^{\circ} \mathrm{C}$ and remains constant with further power increases. The sample heating profile shown in Fig. 2 for etched NSOM probes is qualitatively similar to that mea-

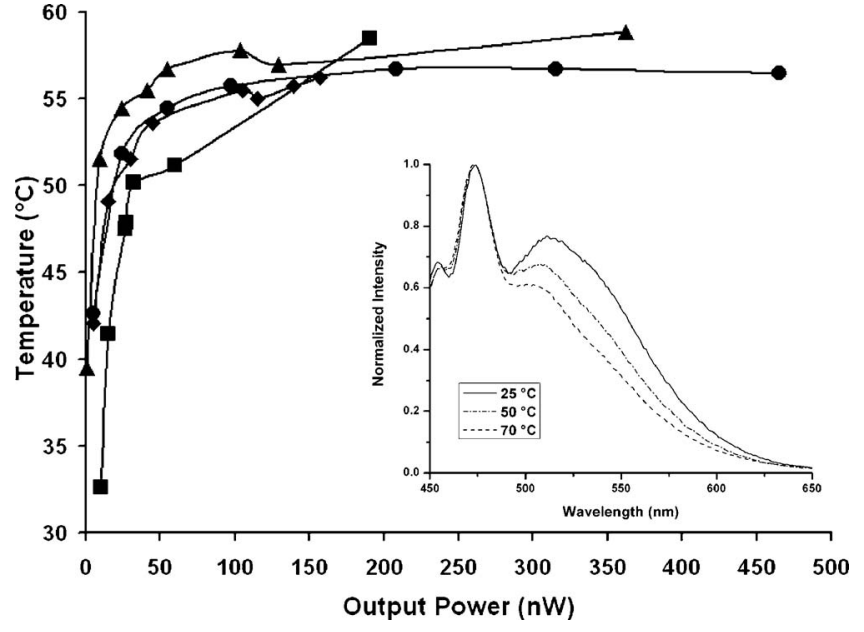

FIG. 2. Plots of sample temperature as a function of output power for several etched NSOM probes. The inset shows the bulk temperature dependent spectra of the thermochromic perylene and $N$-allyl- $N$-methylaniline polymer sample, normalized to the peak at $475 \mathrm{~nm}$.

sured previously with pulled NSOM probes. In that study, similar rises in sample temperature where observed with a maximal sample heating of $55-65{ }^{\circ} \mathrm{C}$ at output powers of $\sim 50 \mathrm{nW}^{18}$

In the previous study, it was suggested that the leveling off effect with increasing power arises from heat induced probe expansion that effectively increases the aperturesample gap at higher powers. Differences in the linear expansion coefficients for aluminum $\left(\alpha=22.2 \times 10^{-6} \mathrm{~K}^{-1}\right)$ and quartz $\left(\alpha=0.59 \times 10^{-6} \mathrm{~K}^{-1}\right)$ result in a differential expansion of the metal coating and dielectric core of the NSOM probe as power is increased. These differences can lead to the effective increase in distance between the NSOM aperture and sample, thus reducing heating at the sample. ${ }^{15,18}$

The sample heating profiles for etched probes shown in Fig. 2 are strikingly similar to those measured with pulled NSOM probes. The longer taper of pulled probes leads to loss farther from the aperture when compared to the smaller aspect ratio etched probes. While this additional loss likely increases heating along the taper of the probe, our results show that this does not significantly increase the sample heating directly under the NSOM aperture. This somewhat unexpected result therefore shows that while etched NSOM
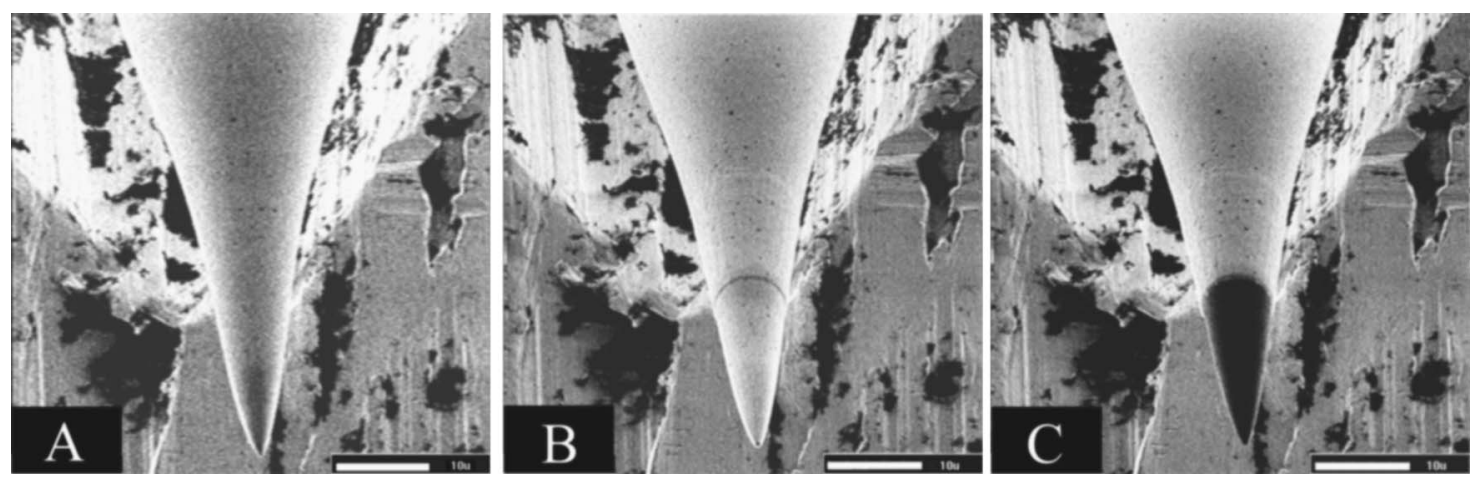

FIG. 3. Series of high resolution images of a pulled NSOM probe as power into the probe is increased. (A) At low powers, the probe appears normal and the aluminum coating is intact. (B) As the NSOM probe temperature increases at elevated output powers, a small stress fracture in the aluminum coating forms approximately $35 \mu \mathrm{m}$ from the aperture of the tip. (C) Above the damage threshold the aluminum coating below the fracture is lost, exposing the nonconductive dielectric which appears dark. The scale bar is $10 \mu \mathrm{m}$. 
probes are vastly more efficient at delivering light, they apparently do not reduce sample heating when compared with pulled NSOM probes. ${ }^{18}$ However, both probe types impart only modest sample heating which suggests that NSOM should be compatible with thermally sensitive samples.

\section{B. NSOM probe failure}

We have also investigated the mechanism of lightinduced NSOM probe failure as increasing power is coupled into both pulled and etched NSOM probes. Fiber optic NSOM probes generally fail at high output powers resulting from the sudden loss of the aluminum coating near the aperture. This has been attributed to either mechanical failure due to shear stresses arising from the different thermal expansion coefficients of the dielectric and metal coating or melting of the aluminum coating. ${ }^{3}$

To understand the mechanisms of failure at elevated output powers, NSOM probes were observed at high resolution while increasing the power to the point of failure. NSOM probes were introduced into the vacuum chamber of a FIB instrument through a custom flange that allowed light to be coupled into the probes. This enables the real time observation of tip failure at elevated powers.

Figure 3 shows a series of images of the same NSOM probe, fabricated using the pulling method, taken as the input power was increased. The series shows the NSOM probe at low powers [Fig. 3(a)] where no damage is observed, at higher powers where damage to the aluminum coating initially appears [Fig. 3(b)], and finally following failure [Fig. 3(c)]. In Fig. 3(b) a fracture is first observed in the aluminum coating approximately $35 \mu \mathrm{m}$ from the aperture, where the diameter of the taper is $\sim 6.5 \mu \mathrm{m}$. This initial fracture eventually leads to the complete removal of aluminum from the region below the fracture as seen in Fig. 3(c). The loss of the aluminum coating from the taper of the probe leads to the dramatic increase in light throughput that signals tip failure.

The stress fracture observed in Fig. 3(b) suggests that damage to the coating arises as a result of shear stresses at elevated heating. The linear expansion of a material is described by

$$
\Delta L=\alpha L_{o} \Delta T,
$$

where $\Delta L$ represents the change in length of the material, $\alpha$ is the linear expansion coefficient for a given material, $L_{o}$ is the original length of the material, and $\Delta T$ is the change in temperature. The large difference in the linear expansion coefficients for aluminum and quartz, $\alpha=22.2 \times 10^{-6} \mathrm{~K}^{-1}$ and $\alpha=0.59 \times 10^{-6} \mathrm{~K}^{-1}$, respectively, leads to a mismatch in expansion during heating. As the temperature of the probe increases, the aluminum coating expands to a greater extent than the dielectric core. Eventually, shear stresses fracture the metal coating around the taper as seen in Fig. 3(b) and lead to the total removal of coating as shown in Fig. 3(c).

For NSOM probes fabricated using chemical etching, the mechanism of probe failure is less clear, but appears to involve melting of the aluminum coating. Figures 4(a) and 4(b) show an etched NSOM probe before and after probe failure, respectively. Unlike the image shown in Fig. 3(b), we have

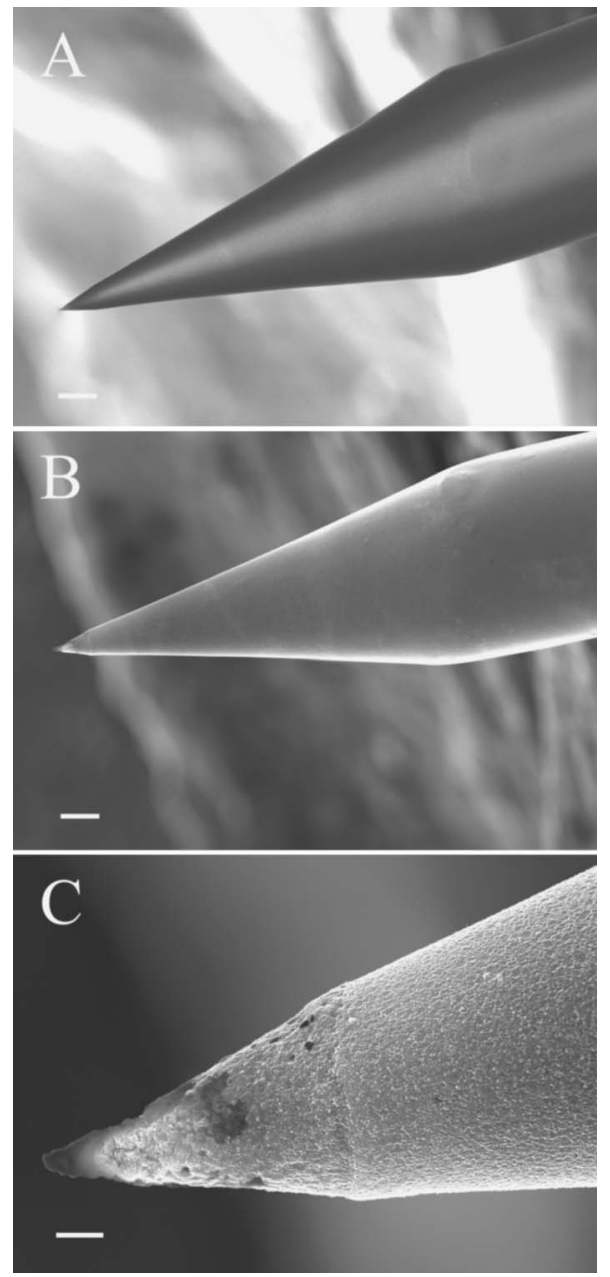

FIG. 4. Series of high resolution images of an etched NSOM probe as power into the probe is increased. Panels (A) and (B) show the etched NSOM probe before and after failure, respectively (scale bar is $20 \mu \mathrm{m}$ ). (C) Magnified view of the NSOM probe following failure (scale bar is $2 \mu \mathrm{m}$ ).

not found any clear evidence for a stress fracture preceding the ultimate failure at the tip apex for etched NSOM probes. The probe failure is rapid once the damage threshold is reached and the resulting removal of the metal coating is less uniform as seen in Fig. 4(b). Moreover, small islands that apparently reflect globular aluminum remain on the probe, possibly suggesting a failure mechanism in which the aluminum coating melts at elevated powers. Previous studies that have measured heating along the taper in pulled NSOM probes have reported heating as high as $470{ }^{\circ} \mathrm{C} .{ }^{8}$ These measurements were conducted $70 \mu \mathrm{m}$ from the aperture and it is feasible that temperatures may approach the melting point of bulk aluminum $\left(660^{\circ} \mathrm{C}\right)$ at distances closer to the aperture of the probe where we find damage. It is also possible that the different surface properties of etched versus pulled probes lead to the differences in metal coating failure observed. The surface of HF etched probes fabricated with the Turner method is rough compared with pulled probes, and surface chemistry differences along with increased surface area may strengthen the metal coating attachment. With stronger adhesion, metal coating failure may not involve the large and complete loss of metal coating as observed for 


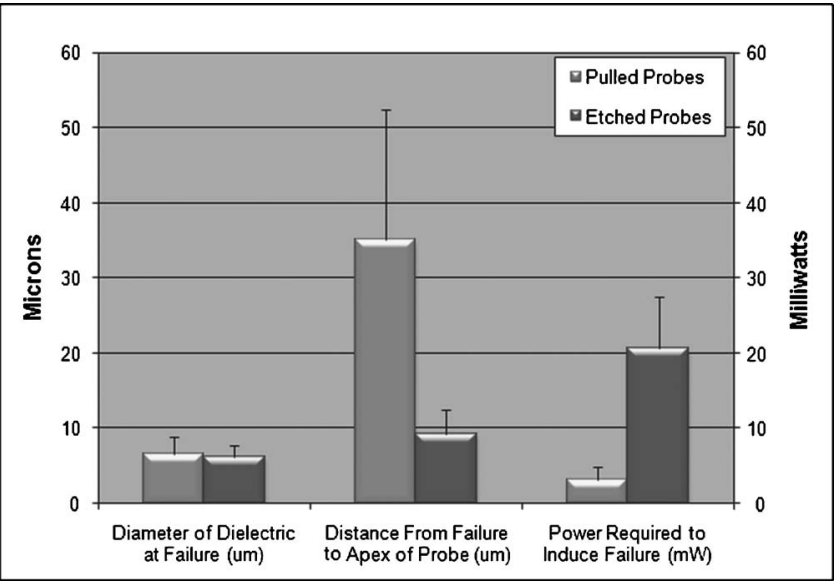

FIG. 5. Comparison of the performance parameters for NSOM probes fabricated with the pulling and etching methods. The data were obtained by combining the results from 12 pulled and 15 etched probes.

pulled probes and may lead to the formation of the observed small islands.

A summary of the high resolution studies of probe failure for both types of NSOM probes are compared in Fig. 5. As seen in Fig. 5, the taper diameter at which probe failure initiates is similar for both etched and pulled NSOM probes as expected once the cutoff diameter is reached. We find that for both types of probes, the aluminum coating is compromised when the taper diameter reaches approximately $6 \mu \mathrm{m}$ in diameter. In Fig. 5, the average distance from the point of failure to the tip apex for pulled probes is $35.2 \pm 17.3 \mu \mathrm{m}$ while it is only $9.2 \pm 3.4 \mu \mathrm{m}$ for the etched probes. The larger taper angle in etched probes supports propagating modes closer to the tip aperture before they become evanescent and decay rapidly. At this point, light lost into the surrounding metal coating heats the probe and eventually leads to failure. ${ }^{3,11}$

There is also a large difference in the power required to cause tip failure in etched versus pulled probes which reflects the difference in taper angle (Fig. 5). For probes fabricated in our laboratory, the input power required to induce probe failure for pulled tips is $\sim 3.4 \pm 1.7 \mathrm{~mW}$, while the power required to cause failure in etched probes is approximately six times greater at $\sim 20.7 \pm 6.9 \mathrm{~mW}$. These input powers represent the actual power into the tip as coupling losses have been accounted for and tested after each measurement. As shown by Eq. (1), the expansion mechanism becomes less important as the length over which the heating occurs is reduced. For etched tips, the large taper angle results in less expansion of the aluminum coating past the dielectric for a given input power, thus increasing the total amount of light that can be delivered before damage.

Varying the half-cone angle of the NSOM probe taper from $6^{\circ}$ for the pulled probes to $15^{\circ}$ for the etched probe can theoretically increase throughput by four to five orders of magnitude. ${ }^{3}$ While etched probes are more efficient and have higher damage thresholds, our results show that their sample heating characteristics are nearly identical to pulled NSOM probes. Results from high resolution studies suggest that the failure mechanisms are different for the two probe geom- etries. For pulled NSOM probes, the thermal expansion of the probe at elevated temperatures leads to shear stresses that damage the metal coating. The shorter length over which heating occurs in etched NSOM probes leads to higher outputs that appear to eventually lead to thermal damage of the metal layer.

\section{SUMMARY}

Sample heating as a function of output power from NSOM probes fabricated using the etching method has been characterized using a thermochromic polymer sample. At low powers, sample heating rises sharply before plateauing at approximately $55-60{ }^{\circ} \mathrm{C}$ with output powers above $\sim 50-100 \mathrm{nW}$. Interestingly, this behavior is nearly identical to heating trends measured for NSOM probes fabricating using the pulling method. These results indicate that only modest sample heating occurs in NSOM measurements using either pulled or etched probes. As power is increased further, probes will fail due to the loss of the metal surrounding the tip aperture. High resolution measurements of NSOM probes fabricated using both approaches suggest that failure occurs through different mechanisms for each. For pulled NSOM probes, differential expansion of the dielectric and metal coating at elevated powers leads to stress fractures in the metal coatings that eventually lead to loss of the metal around the taper of the probe. For etched probes, however, clear stress fractures are not observed and the morphology of the damaged aluminum suggests that melting of the coating may be the source of failure.

\section{ACKNOWLEDGMENTS}

One of the authors (R.C.D.) gratefully acknowledges support from the NIH (GM65964) and the Madison and Lila Self Foundation. Another author (N.E.D.) gratefully acknowledges support from the NIH Dynamic Aspects of Chemical Biology training program (GM008545-13).

${ }^{1}$ R. C. Dunn, Chem. Rev. (Washington, D.C.) 99, 2891 (1999).

${ }^{2}$ M. Edidin, Traffic (Oxford, U. K.) 2, 797 (2001).

${ }^{3}$ L. Novotny and B. Hecht, Principles of Nano-Optics (Cambridge University Press, New York, 2006).

${ }^{4}$ B. N. Flanders and R. C. Dunn, Ultramicroscopy 91, 245 (2002).

${ }^{5}$ P. F. Barbara, D. M. Adams, and D. B. O'Connor, Annu. Rev. Mater. Sci. 29, 433 (1999).

${ }^{6}$ B. Hecht, B. Sick, U. P. Wild, V. Deckert, R. Zenobi, O. J. F. Martin, and D. W. Pohl, J. Chem. Phys. 112, 7761 (2000).

${ }^{7}$ B. I. Yakobson, A. LaRosa, H. D. Hallen, and M. A. Paesler, Ultramicroscopy 61, 179 (1995).

${ }^{8}$ M. Stahelin, M. A. Bopp, G. Tarrach, A. J. Meixner, and I. Zschokke-Granacher, Appl. Phys. Lett. 68, 2603 (1996).

${ }^{9}$ A. Lazarev, N. Fang, Q. Luo, and X. Zhang, Rev. Sci. Instrum. 74, 3679 (2003).

${ }^{10}$ E. Betzig, J. K. Trautman, T. D. Harris, J. S. Weiner, and R. L. Kostelak, Science 251, 1468 (1991).

${ }^{11}$ P. Burgos, Z. Lu, A. Ianoul, C. Hnatovsky, M. L. Viriot, L. J. Johnston, and R. S. Taylor, J. Microsc. 211, 37 (2003).

${ }^{12}$ P. Hoffmann, B. Dutoit, and R.-P. Salathe, Ultramicroscopy 61, 165 (1995).

${ }^{13}$ D. W. Pohl, IBM J. Res. Dev. 39, 701 (1995).

${ }^{14}$ P. G. Gucciardi, M. Colocci, M. Labardi, and M. Allegrini, Appl. Phys. Lett. 75, 3408 (1999). 
${ }^{15}$ C. Lienau, A. Richter, and T. Elsaesser, Appl. Phys. Lett. 69, 325 (1996). ${ }^{16}$ A. H. L. Rosa, B. I. Yakobson, and H. D. Hallen, Appl. Phys. Lett. 67, 2597 (1995).

${ }^{17}$ L. Thiery, N. Marini, J.-P. Prenel, M. Spajer, C. Bainier, and D. Courjon, Int. J. Therm. Sci. 39, 519 (2000)

${ }^{18}$ E. S. Erickson and R. C. Dunn, Appl. Phys. Lett. 87, 201102 (2005).

${ }^{19}$ V. Kurpas, M. Libenson, and G. Martsinovsky, Ultramicroscopy 61, 187 (1995).
${ }^{20}$ L. Thiery and N. Marini, Ultramicroscopy 94, 49 (2003).

${ }^{21}$ D. R. Turner, U.S. Patent No. 4,469,554 (1984).

${ }^{22}$ C. W. Hollars and R. C. Dunn, Rev. Sci. Instrum. 69, 1747 (1998).

${ }^{23}$ N. Chandrasekharan and L. A. Kelly, J. Am. Chem. Soc. 123, 9898 (2001).

${ }^{24}$ C. W. Hollars and R. C. Dunn, J. Chem. Phys. 112, 7822 (2000).

${ }^{25}$ L. Novotny and C. Hafner, Phys. Rev. E 50, 4094 (1994).

${ }^{26}$ P. Moar, F. Ladouceur, and L. Cahill, Appl. Opt. 39, 1966 (2000). 